\title{
Calculate the Quality Measures on Classification of Continuous EEG without Trial Structure EEG Dataset
}

\author{
Mangesh J. Patil \\ Department of CS and IT, \\ Dr. Babasaheb Ambedkar \\ Marathwada University, \\ Aurangabad MS \\ India
}

\author{
Mukta G. Dhopeshwarkar \\ Department of CS and IT, \\ Dr. Babasaheb Ambedkar \\ Marathwada University, \\ Aurangabad MS \\ India
}

\author{
Pankaj A. Sathe \\ Department of CS and IT, \\ Dr. Babasaheb Ambedkar \\ Marathwada University, \\ Aurangabad MS \\ India
}

\begin{abstract}
Quality measure is very significant method for signal processing. Using this processes we can evaluate the EEG signal to see whether the data are noisy or not. The quality measure is performed on BCI competition dataset, this dataset is having 14 EEG signal, $0.05-200 \mathrm{~Hz}, 1000 \mathrm{~Hz}$ sampling rates, 2 classes of 7 subjects. The resultant signal quality is verified by using different quality measures parameters like PSNR, MSE, MAXERR, and L2RAT. So it is conclude that quality of EEG signal has been enriched by using of median filter. Hence it is proved that the recognition rate is increases.
\end{abstract}

\section{General Terms}

Calculate the quality measures (PSNR, MSE, MAXERR, and L2RAT) on classification of continuous EEG without trial structure EEG dataset

\section{Keywords}

Quality measure, PSNR, MSE, MAXERR, L2RAT

\section{INTRODUCTION}

Emotion classification from EEG data has involved much courtesy with the rapid development of dry electrode techniques, machine learning algorithms, and various real-world applications of brain- computer interface for normal people. Researchers had little sympathetic of the details of relationship between different emotional states and various EEG features. To improve the accuracy of EEG-based emotion classification and visualize the changes of emotional states with time [1].

Trait emotional intelligence (trait EI) is a gathering of emotionrelated characters is the process for capturing the level to which people experience, attend to identify, understand, control, and consume their emotions and those of others. According to authors, emotional intelligence might be related with differential frontal activation. The pattern of resting electroencephalographic (EEG) activation recorded in the frontal areas was significantly associated with emotional intelligence. Persons with higher trait EI evidence greater resting left frontal activation [2].

Author introduces an emotion recognition system built on electroencephalogram (EEG) signals. Trials using movie elicitation are intended for obtaining subject's EEG signals to categorize four emotion states: joy, relax, sad, and fear. Subsequently pre-processing the EEG signals, then examine various kinds of EEG features to form an emotion recognition system. To assess classification performance, $\mathrm{k}$ nearest neighbor
(kNN) algorithm, multilayer perceptron and support vector machines were used as classifiers. Experimental results specify that an average test accuracy of $66.51 \%$ for classifying four emotion situations can be obtained by using frequency domain features and support vector machines [3].

Author have implement a multimodal emotion recognition framework by joining eye movements and electroencephalography (EEG) to enhance emotion recognition The two main contributions is present in this research. a) Examine sixteen eye activities related to emotions and identify the essential patterns of these eye movements for three emotional situations: positive, neutral and negative. b) Observe various modality fusion approaches for assimilating users external hidden behaviors and internal mental states and reveal that the characteristics of eye movements and EEG are complementary to emotion recognition. Investigate results prove that modality fusion could expressively improve emotion recognition accuracy in evaluation with single modality. The best accuracy got by fuzzy integral fusion strategy is $87.59 \%$, although the accuracies of solely using eye movements and EEG data are $77.80 \%$ and $78.51 \%$, respectively [4].

Author have done the analysis of a 12-subject electroencephalographic (EEG) data set in which contributors were requested to engage in prolonged, self-paced occurrences of guided emotion imagination with eyes closed. The goal is to acceptably predict, given a short EEG segment, whether the participant was imagining a positive respectively negativevalence emotional situation during the given segment using a predictive model learned via machine learning. Using a variant of the Filter- Bank Common Spatial Pattern algorithm, algorithm achieve an average accuracy of $71.3 \%$ correct classification of binary valence rating across 12 different emotional imagery scenarios under rigorous block-wise cross-validation [5]. The EEG data has been composed from 6 healthy subjects with in an age group of 21-27 using 63 biosensors. From the personal analysis on each emotion, three emotions have been recognized with higher agreement. After preprocessing the signals, discrete wavelet transform is performed to extract the EEG parameters. The feature vectors consequent from the above feature extraction method on 63 biosensors form an input matrix for emotion classification. Authors have used Fuzzy C-Means (FCM) and Fuzzy k-Means (FKM) clustering methods for classifying the emotions. Also analyzed the performance of FCM and FKM on reduced number of 24 biosensors model [6]. 




Figure 1: Workflow of Quality Measures on Classification of Continuous EEG without Trial Structure Dataset

\section{METHODOLOGY}

As portrayed in figure 1, for performing the quality measure of EEG signal, quality measure parameters were use. Like peak signal to noise ratio (PSNR), mean square error (MSE), max error (MAXERR) and L2RAT is the ratio of the squared norm.

PSNR: PSNR is the peak signal to noise ratio in decibels. The PSNR is only significant for data encrypted in terms of bits per sample, or bits per pixel. For example, an image with 8 bits per pixel contains integers from 0 to 255 .

MSE: The mean square error (MSE) is the squared norm of the difference between the data and the approximation divided by the number of elements.

MAXERR: MAXERR is the maximum absolute squared deviation of the data from the approximation.

L2RAT: L2RAT is the ratio of the squared norm of the signal or image approximation to the input signal or image.

Following are the formulas for the quality measure parameters.

$$
P S N R=20 \log _{10}\left(\frac{M A X_{f}}{\sqrt{M S E}}\right)
$$

The mathematical representation of the PSNR is as follows:

$$
\begin{aligned}
& M S E=\frac{1}{m n} \sum_{0}^{m-1} \sum_{0}^{n-1}\|f(i, j)-g(i, j)\|^{2} \\
& M S E=\left(\frac{1}{(m * n)}\right) * \operatorname{sum}\left(\operatorname{sum}\left((f-g) .^{2}\right)\right) \\
& P S N R=\frac{20 * \log (\max (\max (f)))}{\left((M S E)^{0.5}\right)}
\end{aligned}
$$

Where the MSE (Mean Squared Error) is: This can also be represented in a text based format as: Where $f$ represent the matrix data of our original signal. $g$ represents the matrix data of our degraded signal. $m$ represents the numbers of rows of value of the EEG signal and $i$ represents the index of that row. $n$ represents the number of columns of value of the EEG signal and $j$ represents the index of that column. MAXRR is the maximum signal value that exists in our original "known to be good" image. The mean square error or MSE of an estimator is one of many ways to compute the difference between an estimator and the true value of the quantity being estimated. MSE is a risk function, corresponding to the expected value of the squared error loss or quadratic loss. MSE measures the average of the square of the "error." The error is the amount by which the estimator differs from the quantity to be estimated. The difference occurs because of randomness or because the estimator doesn't account for information that could produce a more accurate estimate. The mean squared error (MSE) for our practical purposes allows us to compare the "true" pixel values of our original image to our degraded image. The MSE represents the average of the squares of the "errors" between our actual image and our noisy image. The error is the amount by which the values of the original image differ from the degraded image. The proposal is that the higher the PSNR, the better degraded image has been reconstructed to match the original image and the better the reconstructive algorithm. This would occur because to minimize the MSE between images with respect the maximum signal value of the image The Mean Squared Error (MSE) is a measure of how close a fitted line is to data points. For every data point, you take the distance vertically from the point to the corresponding y value on the curve fit, this is known as the error, and square the value. Next you add up all those values for all data points, and divide by the number of points. The reason for squaring is so negative values do not 
cancel positive values. The smaller the Mean Squared Error, the closer the fit is to the data. The MSE has the units squared of whatever is plotted on the vertical axes. The MSE of an estimator with respect to the estimated parameter $\theta$ is defined as

$$
\operatorname{MSE}(\hat{\theta})=E\left[\hat{\theta}-\theta^{2}\right]
$$

The MSE is equal to the sum of the variance and the squared bias of the estimator

The MSE thus assesses the quality of an estimator in terms of its variation and unbiasedness. Note that the MSE is not equivalent to the expected value of the absolute error. MSE is an expectation; it is a scalar, and not a random variable. It may be a function of the unknown parameter $\theta$, but it does not depend on any random quantities. However, when MSE is

$$
\operatorname{MSE}(\hat{\theta})=\operatorname{var}(\hat{\theta})+(\operatorname{Bias}(\hat{\theta}-\theta))
$$

computed for a particular estimator of $\theta$ the true value of which is not known, it will be subject to estimation error. In a Bayesian sense, this means that there are cases in which it may be treated as a random variable.

Maxerr is the maximum absolute squared deviation of the

$$
S=\sum_{i=1}^{n} f\left(y_{i}\right)\left(x_{i}-x_{i-1}\right)
$$

L2RAT is the ratio of the squared norm of the signal or image approximation to the input signal. Table 1 and Table 2 shows the output of EEG signal after performing the quality measures.

\section{RESULT}

Quality measure of EEG data is done by using PSNR, MSE, MAXERR and L2RAT. Quality measure is very important task in EEG signal processing, because at the time of signal acquiring the noise may get added due to unwanted situations. Using MATLAB software, we performed the quality measure of original EEG signal followed by processed EEG signal (median filter). Median filter is applied for noise cancelation of EEG data

Table 1: Quality measure of original EEG Signal

\begin{tabular}{|c|c|c|c|c|c|}
\hline $\begin{array}{c}\text { Sr. } \\
\text { No }\end{array}$ & Name of EEG File & PSNR & MSE & MAXERR & L2RAT \\
\hline 1 & & & & & \\
\hline 2 & BCICIV_calib_ds1a & -87.1324 & $3.3599 \mathrm{e}+13$ & $3.3369 \mathrm{e}+04$ & $1.0432 \mathrm{e}+08$ \\
\hline 3 & BCICIV_calib_ds1b & -76.5912 & $2.9662 \mathrm{e}+12$ & $6.9810 \mathrm{e}+03$ & $2.8632 \mathrm{e}+07$ \\
\hline 4 & BCICIV_calib_ds1c & -80.3742 & $7.0877 \mathrm{e}+12$ & $5.7944 \mathrm{e}+03$ & $2.3406 \mathrm{e}+10$ \\
\hline 5 & BCICIV_calib_ds1d & -74.8246 & $1.9749 \mathrm{e}+12$ & $5.2783 \mathrm{e}+03$ & $1.2282 \mathrm{e}+10$ \\
\hline 6 & BCICIV_calib_ds1e & -66.0228 & $2.6023 \mathrm{e}+11$ & $2.2242 \mathrm{e}+03$ & $1.8097 \mathrm{e}+11$ \\
\hline 7 & BCICIV_calib_ds1f & -79.5143 & $5.8145 \mathrm{e}+12$ & $2.4666 \mathrm{e}+04$ & $2.5211 \mathrm{e}+07$ \\
\hline 8 & BCICIV_eval_ds1a & -75.8482 & $2.4998 \mathrm{e}+12$ & $4.3046 \mathrm{e}+03$ & $4.1166 \mathrm{e}+07$ \\
\hline 9 & BCICIV_eval_ds1b & -77.7592 & $3.8815 \mathrm{e}+12$ & $1.4481 \mathrm{e}+04$ & $3.1865 \mathrm{e}+07$ \\
\hline 10 & BCICIV_eval_ds1c & -63.0355 & $1.3081 \mathrm{e}+11$ & $2.5868 \mathrm{e}+03$ & $2.6741 \mathrm{e}+10$ \\
\hline 11 & BCICIV_eval_ds1d & -69.3952 & $5.6572 \mathrm{e}+11$ & $4.7814 \mathrm{e}+03$ & $1.9773 \mathrm{e}+10$ \\
\hline 12 & BCICIV_eval_ds1e & -66.5032 & $2.9067 \mathrm{e}+11$ & $2.1968 \mathrm{e}+03$ & $5.1554 \mathrm{e}+11$ \\
\hline 13 & BCICIV_eval_ds1f & -80.4389 & $7.1941 \mathrm{e}+12$ & $2.9673 \mathrm{e}+04$ & $3.3837 \mathrm{e}+07$ \\
\hline 14 & BCICIV_eval_ds1g & -79.8930 & $6.3443 \mathrm{e}+12$ & $1.2070 \mathrm{e}+04$ & $3.0872 \mathrm{e}+07$ \\
\hline
\end{tabular}

Table 2: Quality measure of processed (median filter) EEG Signal

\begin{tabular}{|c|c|c|c|c|c|}
\hline $\begin{array}{c}\text { Sr. } \\
\text { No }\end{array}$ & Name of EEG File & PSNR & MSE & MAXERR & L2RAT \\
\hline 1 & BCICIV_calib_ds1a & -0.0900 & $6.6387 \mathrm{e}+04$ & 15451 & 1.0123 \\
\hline 2 & BCICIV_calib_ds1b & 2.3818 & $3.7575 \mathrm{e}+04$ & 4780 & 1.0688 \\
\hline 3 & BCICIV_calib_ds1c & -0.1147 & $6.6765 \mathrm{e}+04$ & 3075 & 1.1818 \\
\hline 4 & BCICIV_calib_ds1d & -0.7375 & $7.7061 \mathrm{e}+04$ & 4468 & 2.5893 \\
\hline 5 & BCICIV_calib_ds1e & 10.9843 & $5.1838 \mathrm{e}+03$ & 2075 & 1.3452 \\
\hline
\end{tabular}




\begin{tabular}{|c|c|c|c|c|c|}
\hline 6 & BCICIV_calib_ds1f & -3.3140 & $1.3947 \mathrm{e}+05$ & 23810 & 1.1875 \\
\hline 7 & BCICIV_calib_ds1g & 1.8833 & $4.2146 \mathrm{e}+04$ & 6368 & 1.0493 \\
\hline 8 & BCICIV_eval_ds1a & 5.0019 & $2.0554 \mathrm{e}+04$ & 2708 & 1.1016 \\
\hline 9 & BCICIV_eval_ds1b & 1.8309 & $4.2657 \mathrm{e}+04$ & 14198 & 1.0910 \\
\hline 10 & BCICIV_eval_ds1c & 10.9652 & $5.2067 \mathrm{e}+03$ & 2240 & 2.5562 \\
\hline 11 & BCICIV_eval_ds1d & 3.1380 & $3.1571 \mathrm{e}+04$ & 4806 & 5.5347 \\
\hline 12 & BCICIV_eval_ds1e & 11.4263 & $4.6822 \mathrm{e}+03$ & 1989 & 1.3507 \\
\hline 13 & BCICIV_eval_ds1f & -4.7817 & $1.9555 \mathrm{e}+05$ & 27956 & 1.2591 \\
\hline 14 & BCICIV_eval_ds1g & -2.4615 & $1.1461 \mathrm{e}+05$ & 8730 & 1.2200 \\
\hline
\end{tabular}



(A)

Figure 2: (A) Quality Measure of Original Signal and

\section{CONCLUSION}

Proposed algorithm is developed for quality measure of continuous EEG without trial structure EEG dataset. In this whole process, we have performed the quality measure (PSNR, MSE, MAXERR \& L2RAT) of EEG signal. For evaluation of this algorithm, we have collected the EEG data from BCI Competition resources. Then process this EEG data for calculating the quality measure of the collected signal using MATLAB software. According to the table 1 and 2, we can conclude that the processed signals are more robust as compare to original EEG signal.

\section{ACKNOWLEDGMENTS}

We are thankful to Benjamin Blankertz, Carmen Vidaurre, Celmens Brunner and Robert Leeb for providing BCI competition IV dataset.

\section{REFERENCES}

[1] Xiao-Wei Wang, Dan Nie, Bao-Liang Lu, "Emotional state classification from EEG data using machine learning approach", ScienceDirect, Neurocomputing 129 (2014) 94106.

[2] Moïra Mikolajczak, Kerrin Bodarwé, Olivier Laloyaux, Michel Hansenne, Delphine Nelis, "Association between frontal EEG asymmetries and emotional intelligence among adults", ScienceDirect Personality and Individual Differences, 2009.

\section{Processed Signal}

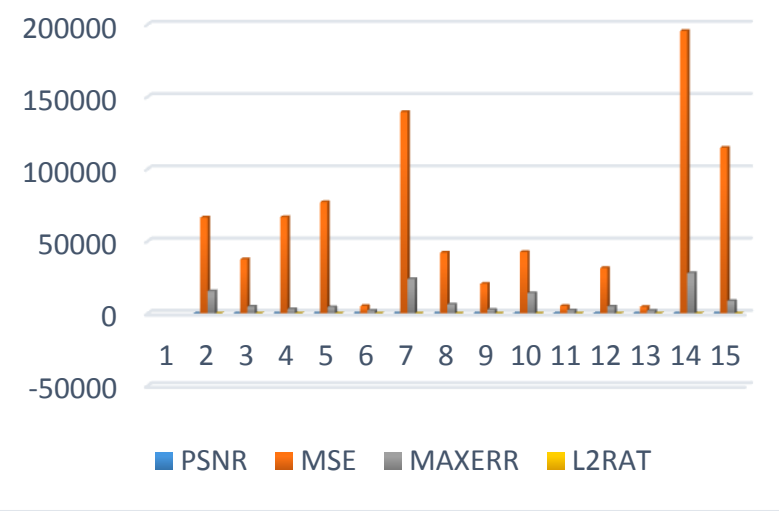

(B)

[3] Xiao-Wei Wang, Dan Nie, and Bao-Liang Lu, "EEG-Based Emotion Recognition Using Frequency Domain Features and Support Vector Machines", ICONIP 2011, L N C S 7062, Springer-Verlag Berlin Heidelberg 2011.

[4] Yifei Lu1, Wei-Long Zheng, Binbin Li1, and Bao-Liang $\mathrm{Lu}$, "Combining Eye Movements and EEG to Enhance Emotion Recognition", Proceedings of the Twenty-Fourth International Joint Conference on Artificial Intelligence (IJCAI) 2015 .

[5] Christian Andreas Kothe, Scott Makeig, and Julie Anne Onton, "Emotion Recognition from EEG During Self-Paced Emotional Imagery", Kothe, C., Onton, J., Makeig, S. "Emotion Recognition from EEG During Self-Paced Emotional Imagery." In proceedings of: Affective BrainComputer Interfaces (aBCI) Workshop, IEEE Affective Computing and Intelligent Interaction 2013, Geneva Switzerland, 2013.

[6] M. Murugappan, M. Rizon, R. Nagarajan, S. Yaacob, I. Zunaidi, and D. Hazry, "EEG Feature Extraction for Classifying Emotions using FCM and FKM", International Journal Of Computers And Communications Issue 2, Volume 1, 2007.

[7] http://www.bbci.de/competition/iv date of access 25-062016. 